\title{
Editorial
}

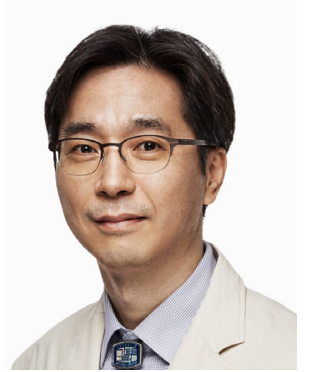

Seung Joon Kim

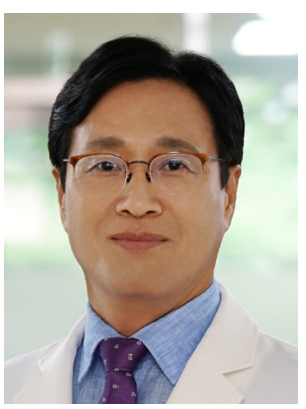

Kyung-Sun Kang

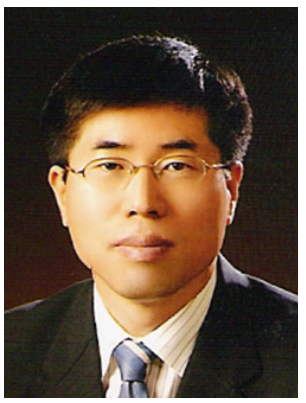

Hyung-Ryong Kim

Received: December 15, 2020

Accepted: January 1, 2021

Correspondence to:

Seung Joon Kim, MD, PhD

Department of Internal Medicine, St.

Mary's Hospital, Postech-Catholic

Biomedical Engineering Institute,

College of Medicine, The Catholic

University of Korea, 222 Banpo-daero,

Seocho-gu, Seoul 06591, Korea

E-mail: cmcksj@catholic.ac.kr

\section{Welcome to Organoid: a new and innovative journal}

\author{
SeungJoon Kim ${ }^{1}$, Kyung-Sun Kang ${ }^{2}$, Hyung-Ryong Kim ${ }^{3}$ \\ ${ }^{1}$ Department of Internal Medicine, St. Mary's Hospital, Postech-Catholic Biomedical Engineering \\ Institute, College of Medicine, The Catholic University of Korea, Seoul, Korea \\ ${ }^{2}$ Adult Stem Cell Research Center, College of Veterinary Medicine, Seoul National University, Seoul, \\ Korea \\ ${ }^{3}$ College of Dentistry and Institute of Tissue Regeneration Engineering, Dankook University, Cheonan, \\ Korea
}

We are very pleased to announce a new international journal, Organoid. This journal is an official publication of the Organoid Society. Organoid is supported by the Organoid Society, but it welcomes submissions from authors worldwide.

Although Organoid is currently in its earliest stages as a journal, we aim to publish numerous articles, as the field of organoid research is rapidly expanding and attracting the attention of researchers throughout the world. Through our online manuscript management system, we provide high standards of support for authors and reviewers. We anticipate that Organoid will dynamically evolve and appropriately respond to the changing needs of the research field. We hope that Organoid, as an emerging journal, will open the door for future explorations in organoid research.

The journal's mission is to share insights and developments in organoid research with a global audience. The long-term vision of the journal is to publish high-quality basic, translational, and biotechnological research in the field of organoids. By doing so, Organoid aims to enhance this research field, which has a wide range of applications, such as modeling organ development and disease, drug discovery, tissue engineering, personalized treatment, and regenerative medicine, which are physiologically relevant and amenable to real-life applications with implications for the well-being of the global community.

Although there are already a number of journals that focus on basic, translational, and biotechnological stem cell research, the need has arisen to publish papers facilitating the application of highly promising scientific discoveries in the field of organoid research. Organoid will stand apart from ordinary stem cell journals by accepting and prioritizing the publication of new and innovative information, rather than conventional results.

Organoid is a peer-reviewed, open-access journal dedicated to publishing articles in the field of organoid research. It will publish original articles, reviews, research methods, analyses, case reports, letters to the editor, and editorials. Case reports will be published, but their number will be limited in each issue. In the issues of Organoid, you will discover thought-provoking articles on new and innovative organoid research.

We have established an Editorial Board comprising an international group of outstanding scientists to meet global standards for the process of peer-reviewing submitted papers. We will try to complete 
the review process and inform authors of the status of their manuscripts within 4 weeks of submission. I am confident that Organoid will grow and receive wide recognition in the near future.

On behalf of Organoid, we hope that you enjoy reading the articles and consider submitting your own valuable work to this exciting new journal. Through collaboration among the Editorial Board members and the associate editors, we will strive to make this journal a high-standard, internationally renowned journal as early as possible. We are confident that Organoid will become a highly trusted leading journal in the field of organoid research.

\section{Notes}

\section{Conflict of interest}

No potential conflict of interest relevant to this article was reported. 